\title{
Dynamic Changes of Lipid Profile in Severe Hypertriglyceridemia-Induced Acute Pancreatitis Patients Under Double Filtration Plasmapheresis: A Retrospective Observational Study
}

\section{Huijun Zheng}

Zhejiang University School of Medicine Sir Run Run Shaw Hospital

\section{Donghai Wang}

Zhejiang University School of Medicine Sir Run Run Shaw Hospital

\section{Xiaoling Wang}

Zhejiang University School of Medicine Sir Run Run Shaw Hospital

\section{Yongjun Lin}

Zhejiang University School of Medicine Sir Run Run Shaw Hospital

\section{Zhihua Lu}

Zhejiang University School of Medicine Sir Run Run Shaw Hospital

\section{Yueliang Chen}

Zhejiang University School of Medicine Sir Run Run Shaw Hospital

\section{Guo Feng}

Zhejiang University School of Medicine Sir Run Run Shaw Hospital

Na Yang ( $\nabla 318023 @ z j u . e d u . c n)$

Zhejiang University School of Medicine Sir Run Run Shaw Hospital

\section{Research}

Keywords: Severe hypertriglyceridemia-induced acute pancreatitis, Double filtration plasma apheresis, Lipid profile, High-density lipoprotein

Posted Date: June 18th, 2020

DOl: https://doi.org/10.21203/rs.3.rs-36064/v1

License: (c) (1) This work is licensed under a Creative Commons Attribution 4.0 International License. Read Full License 
Version of Record: A version of this preprint was published on September 15th, 2020. See the published version at https://doi.org/10.1186/s12944-020-01383-8. 


\section{Abstract}

Background: In this study, we aimed to investigate the dynamic changes of lipid profile under double filtration plasmapheresis (DFPP) in severe hypertriglyceridemia-induced acute pancreatitis (sHTGP) patients and ascertain whether these changes were associated with the prognosis of the disease.

Methods: This retrospective study was conducted at a tertiary critical care center in China. We included sHTGP patients who were admitted within $72 \mathrm{~h}$ after AP onset. All the sHTGP patients received DFPP within $24 \mathrm{~h}$ after admission. Lipid profile changes was measured in 47 patients with sHTGP on admission, consecutive 4 days after DFPP and at discharge. According to the TG levels in the first day after DFPP, we divided patients into two group: non-target ( $T G>5.6 \mathrm{mmol} / \mathrm{L}$ ) and target group ( $\mathrm{TG} \leq 5.6 \mathrm{mmol} / \mathrm{L})$. Propensity score matching was used to investigate association between TG-lowering effect and the prognosis. Linear regression analysis on four models was used to determine the relationship between other lipid parameters and the outcome.

Results: Except for low density lipoprotein, all the parameters of lipid profile at disease onset showed significant difference from their normal levels. The median serum triglyceride (TG) level of the sHTGP patients was $42.9 \mathrm{mmol} / \mathrm{L}$ on admission. In the first day after DFPP, serum TG, cholesterol (TC) and very low density lipoprotein decreased significantly, and the high-density lipoprotein (HDL) as well as apoprotein A1 increased meanwhile. TG continued to fall for the following three days and the other indicators held steady. After propensity score matching, the hospitalization duration was significantly lower in the target group $(14.5[13.0,16.0]$ vs $8.0[6.0,15.3], P=0.035)$. Linear regression analysis revealed that the levels of HDL were negatively associated with the duration of hospitalization in three adjusted models.

Conclusion: There was distinct fluctuation of the lipid profile upon the burst of sHTGP and the parameters changed significantly in the first day after DFPP. Among these parameters, HDL may serve as a biomarker for disease prognosis in patients with sHTGP. Moreover, reaching the TG-lowering target of $5.6 \mathrm{mmol} / \mathrm{L}$ may shorten hospitalization duration.

\section{Background}

Acute pancreatitis (AP) is an inflammatory disease of the pancreas with a worldwide incidence of 13-80 cases per 100,000 per annum [1, 2]. As a common metabolic disorder, severe hypertriglyceridemia (sHTG), defined as a serum triglyceride (TG) level $>11.3 \mathrm{mmol} / \mathrm{L}(1000 \mathrm{mg} / \mathrm{dL})$, is a well-recognized cause of AP accounting for $10-20 \%$ of all cases $[3,4]$. The occurrence of severe hypertriglyceridemia-induced acute pancreatitis (sHTGP) has increased gradually recent decades especially in China [5]. Both primary and secondary disorders of lipoprotein metabolism may be associated with the development of sHTGP [6]. It has been found that metabolic changes of the lipid profile are common in the context of AP and may be associated with the disease prognosis. It was generally considered that the elevated TG level on admission could exacerbate the severity and prognosis of sHTGP [7-9]. In addition, the relationship 
between other parameters of lipid profile such as high density lipoprotein (HDL) and AP has gained more and more attention $[10,11]$. However, the dynamic changes of lipid profile during sHTGP has not been well described.

Lowering TG levels to a safe range rapidly and efficiently is essential for the management of sHTGP patients. A series studies suggested that TG levels $<5.6 \mathrm{mmol} / \mathrm{L}$ represents a safe therapeutic target [12]. However, treatment based on fasting and drugs only is unsatisfactory to achieve a rapid response. Plasmapheresis is considered to be the most effective therapy for lowering TG rapidly in the setting of sHTGP [5]. Double filtration plasmapheresis (DFPP) is one of the options for apheresis [13]. It is a semiselective method based on a double filter system which can selectively remove macromolecules and reduce the use of substitution fluid, such as albumin, compared to other apheresis methods [14]. As far as we known, there is no study reporting the dynamic changes of lipid profile under DFPP in patients with sHTGP by far. In this study, we aimed to characterize dynamic lipid profile changes in sHTGP patients after DFPP, and ascertain whether these changes were associated with the clinical prognosis.

\section{Methods}

\section{Patients}

This was a retrospective observational study conducted in the Department of Critical Care Medicine of Sir Run Run Shaw Hospital (a university-affiliated hospital in Hangzhou, China). The study was approved by the Ethics Committee of Sir Run Run Shaw Hospital (20190215-3). Informed consent from individuals was waived due to the retrospective nature of the study. The study included consecutive patients with sHTGP (admission TG $>11.3 \mathrm{mmol} / \mathrm{L}$ ) who were admitted to the center within 72 hours after AP onset from January 2019 to February 2020. The diagnostic criteria of AP were in accordance with the 2012 revision of the Atlanta classification [15]. Exclusion criteria were as follows: age < 18 years old, pregnancy, complicated with malignant tumor and incomplete information.

\section{Intervention}

According to the clinical practice of our center, all the sHTG patients were suggested to receive DFPP within $24 \mathrm{~h}$ after admission. The DFPP was performed via femoral double lumen using Plasauto EZ machine machine (Asahi-Kasei, Tokyo, Japan), which was loaded with a blood cell separator column (Plasmaflo, OP-08) and a plasma separator (Cascadeflo, EC-40W). Large molecular weight components such as VLDL are discarded. Non-viscous and clean plasma was returned together with the separated cellular components to patient blood circulation. The frequencies of plasmapheresis sessions were determined by the clinicians in each case. Anticoagulation was achieved with heparin.

\section{Data collection}

Baseline characteristics included demographic data, body mass index (BMI), co-morbidities, disease severity, Acute Physiology and Chronic Health Evaluation II score (APACHE II) and Sequential Organ Failure Assessment score (SOFA). Clinical data during hospitalization were also collected, including 
laboratory parameters, systemic complications, length of intensive care unit (ICU) and hospital stay. A lipid profile analysis was collected in each patient on admission, consecutive 4 days after DFPP and at discharge. Lipid profile included serum TG, cholesterol (TC), very low density lipoprotein (VLDL), low density lipoprotein (LDL), HDL and Apoprotein A1 (Apo A1). The primary outcome was hospitalization duration.

\section{Statistical analysis}

Continuous variables with normality distribution were presented as mean \pm SD and analyzed using $t$ test. For variables without normal distribution, continuous variables were presented as median $(25 \%, 75 \%$ interquartile ranges) and Mann-Whitney test was performed. Categorical variables were presented as percentages and analyzed with the $\chi 2$ test or Fisher's exact test. Having met all assumptions, univariate and multivariate linear regression analyses was applied to investigate the association between parameters of lipid profile and length of hospitalization. Four models were used in the linear regression analyses: model 1 was performed as an adjusted model; model 2 was adjusted for age, sex and BMl; model 3 was adjusted for age, sex BMI and APACHE II score; model 4 was adjusted for age, sex BMI, APACHE II score and co-morbidities. The statistical analysis was performed using SPSS 23.0 and Graphpad Prism 6.0. Two-tailed $P$ values $<0.05$ were considered statistically significant.

\section{Results}

\section{Baseline and clinical characteristics of patients with sHTGP}

Data on 47 patients were included in this study. The baseline characteristics are listed in Table 1. The median days from AP onset to admission was 1 and the median serum TG on admission was $42.9 \mathrm{mmol} / \mathrm{L}$ (minimum: $12.8 \mathrm{mmol} / \mathrm{L}$, maximum: $190.9 \mathrm{mmol} / \mathrm{L}$ ). $24 \%$ patients were diagnosis as severe acute pancreatitis (SAP) according to the 2012 Atlanta classification. $36.1 \%$ patients had a history of diabetes. 48.9\% patients had recurrent AP (recurrence times: 2 [1, 3]). According to whether sHTGP patients had AP recurrence, we divided them into non-recurrence and recurrence group. There were no significant differences in baseline and clinical characteristics, except diabetes. The proportion of patients with diabetes was significantly higher in the recurrence group, suggesting diabetes may be a risk factor for $\mathrm{SHTGP}$ recurrence ( $52 \%$ vs $21 \%, P=0.025)$. 
Table 1

Baseline and clinical characteristics of the sHTGP patients

\begin{tabular}{|lllll|}
\hline Variables & $\begin{array}{l}\text { All patients } \\
(\mathbf{n = 4 7 )}\end{array}$ & $\begin{array}{l}\text { non-recurrence } \\
(\mathbf{n}=\mathbf{2 4})\end{array}$ & $\begin{array}{l}\text { recurrence } \\
(\mathbf{n}=\mathbf{2 3})\end{array}$ & $\boldsymbol{P}$ \\
\hline Age, mean $\pm \mathrm{SD}, \mathrm{y}$ & $37.7 \pm 9.6$ & $37.2 \pm 7.9$ & $38.2 \pm 11.3$ & 0.713 \\
\hline Male, $\mathrm{n}(\%)$ & $32(68)$ & $14(58)$ & $18(78)$ & 0.143 \\
\hline BMI, mean \pm SD, kg/m² & $26.9 \pm 3.4$ & $25.3 \pm 4.0$ & $27.7 \pm 2.7$ & 0.338 \\
\hline Disease severity, $\mathrm{n}(\%)$ & & & & 0.897 \\
\hline Mild & $27(57.4)$ & $14(58.4)$ & $13(56.5)$ & \\
\hline Moderate & $9(19.2)$ & $5(20.8)$ & $4(17.4)$ & \\
\hline Severe & $11(23.4)$ & $5(20.8)$ & $6(26.1)$ & \\
\hline APACHE II, median (ranges) & $8.0(6.0,13.0)$ & $7.5(6.0,14.3)$ & $8.0(5.5,14.3)$ & 0.927 \\
\hline SOFA, median (ranges) & $2.0(1.0,3.0)$ & $2.0(1.0,3.0)$ & $2.0(1.0,3.0)$ & 0.981 \\
\hline Co-morbidities, $\mathrm{n}$ (\%) & & & & \\
\hline Hypertension & $8(2.4)$ & $4(16.7)$ & $4(17.4)$ & 0.947 \\
\hline Diabetes & $17(36.1)$ & $5(20.8)$ & $12(52.2)$ & 0.025 \\
\hline ICU days, median (ranges) & $3(2,7)$ & $5(1,7)$ & $3(2,6)$ & 0.914 \\
\hline Hospital days, median (ranges) & $12(8,16)$ & $14(9,16)$ & $10(7,15)$ & 0.105 \\
\hline
\end{tabular}

\section{Dynamic changes in parameters of lipid profile for SHTGP patients}

All the sHTGP patients received DFPP within $24 \mathrm{~h}$ after admission. It was found that serum TG level dropped by an average of $71.2 \%$ in the first day after DFPP for all the patients, of which $36.2 \%$ fell below the safety level $5.6 \mathrm{mmol} / \mathrm{L}$. The level of TG continued to decline significantly in next 3 days and stalled by day 4 (Fig. 1a). Finally, there were $78.7 \%$ patients whose TG level was under $5.6 \mathrm{mmol} / \mathrm{L}$ at discharge. The trend analyses of other lipid profile indicators were conducted meanwhile. The levels of TC and VLDL were higher than the normal range in all the sHTGP patients on admission. Their values decreased significantly after DFPP in day 1 and kept stable in the following days (Fig. 1b-c). The HDL level in $89 \%$ patients was lower than normal range on admission and the values increased significantly after DFPP in day 1 (Fig. 1e). As the major component of HDL, Apo A1 showed the same tendency as HDL (Fig. 1f). Interestingly, serum LDL maintained within normal range in $94 \%$ patients and had no change after DFPP (Fig. 1d). All the patients had resumed oral diet at discharge and their lipid profile showed distinct difference from the admission data. The levels of TG, TC and VLDL at discharge were significantly lower 
than the values on admission, while the levels of HDL and Apo A1 at discharge were significantly higher than admission (Fig. 1).

\section{Association between TG-lowering efficiency and clinical results}

It is generally accepted that control of TG level to well below $5.67 \mathrm{mmol} / \mathrm{L}(500 \mathrm{mg} / \mathrm{dL})$ appear to hold prognostic value. To figure out the effect of DFPP on patients' prognosis, we divided subjects into two groups according to the first day TG values after DFPP: target group ( $T G<5.67 \mathrm{mmol} / \mathrm{L}$ ) and non-target group ( $T G \geq 5.67 \mathrm{mmol} / \mathrm{L}$ ). Considering the influence of initial TG level on prognosis, we performed a propensity score matching. Patients were matched according to age, sex, BMI, APCHE II, SOFA and initial TG level. Finally, 10 patients were matched in the non-target and target group respectively (Table 2). The ICU days for non-target group were longer than target group with no statistical difference $(P=0.089)$. Compared to non-target group, the target group had significantly shorter hospital days $(P=0.035)$.

Table 2

Baseline and outcome of matched sHTGP patients

\begin{tabular}{|llll|}
\hline Variables & Non-target $(\mathbf{n}=\mathbf{1 0})$ & Target $(\mathbf{n}=10)$ & $\boldsymbol{P}$ \\
\hline Age, mean \pm SD, y & $38.6 \pm 7.1$ & $39.0 \pm 9.4$ & 0.930 \\
\hline Male, $\mathrm{n}(\%)$ & $9(90)$ & $6(60)$ & 0.121 \\
\hline BMI, mean \pm SD, $\mathrm{kg} / \mathrm{m}^{2}$ & $27.5 \pm 3.7$ & $26.7 \pm 3.6$ & 0.624 \\
\hline APACHE II, median (ranges) & $7(4,12)$ & $6(4,11)$ & 0.824 \\
\hline SOFA, median (ranges) & $1.5(0.3,2.8)$ & $2(1.0,2.3)$ & 0.783 \\
\hline Serum TG, mean \pm SD, mmol/L & $31.7 \pm 13.0$ & $32.8 \pm 17.1$ & 0.870 \\
\hline ICU days, median (ranges) & $6.5(3.3,7.5)$ & $1.5(1.0,4.3)$ & 0.089 \\
\hline Hospital days, median (ranges) & $14.5(13.0,16.0)$ & $8.0(6.0,15.3)$ & 0.035 \\
\hline
\end{tabular}

To explore the relationship between other lipid profile parameters and clinical results, we performed linear regression analyses based on four models. The results showed that the decrease in serum HDL on admission was significantly associated with the prolonged hospitalization in three models $(P=0.043, P=$ $0.029, P=0.025$ for model $1,3,4$ respectively). APACHE II had a significant association with hospitalization in model 2, 3 and 4 . However, the length of hospitalization was not significantly associated with TC, VLDL, LDL and Apo A1 on admission (Table 3). We also analyzed the relationship between length of hospitalization and lipid profile after DFPP in day 1 and found no significance (data not shown). 
Table 3

Linear regression analyses for parameters of lipid profile with hospital days

\begin{tabular}{|c|c|c|c|c|c|}
\hline \multirow[t]{2}{*}{ Parameters } & \multirow[t]{2}{*}{ model } & \multirow[t]{2}{*}{$\beta$} & \multicolumn{2}{|l|}{$95 \% \mathrm{Cl}$} & \multirow[t]{2}{*}{$P$} \\
\hline & & & lower & upper & \\
\hline \multirow[t]{4}{*}{$\mathrm{TC}$} & 1 & -0.039 & -0.617 & 0.475 & 0.795 \\
\hline & 2 & -0.004 & -0.622 & 0.606 & 0.979 \\
\hline & 3 & -0.051 & -0.722 & 0.535 & 0.764 \\
\hline & 4 & -0.101 & -0.833 & 0.459 & 0.561 \\
\hline \multirow[t]{4}{*}{ VLDL } & 1 & 0.037 & -0.482 & 0.617 & 0.805 \\
\hline & 2 & 0.069 & -0.476 & 0.728 & 0.674 \\
\hline & 3 & 0.002 & -0.608 & 0.616 & 0.990 \\
\hline & 4 & -0.041 & -0.705 & 0.553 & 0.808 \\
\hline \multirow[t]{4}{*}{ LDL } & 1 & -0.038 & -2.195 & 1.706 & 0.802 \\
\hline & 2 & -0.038 & -2.291 & 1.790 & 0.806 \\
\hline & 3 & -0.075 & -2.663 & 1.649 & 0.637 \\
\hline & 4 & -0.078 & -2.694 & 1.647 & 0.628 \\
\hline \multirow[t]{4}{*}{$\mathrm{HDL}$} & 1 & -0.297 & -13.783 & -0.236 & 0.043 \\
\hline & 2 & -0.314 & -14.939 & 0.117 & 0.053 \\
\hline & 3 & -0.346 & -15.370 & -0.882 & 0.029 \\
\hline & 4 & -0.356 & -15.634 & -1.128 & 0.025 \\
\hline \multirow[t]{4}{*}{ Apo A1 } & 1 & -0.058 & -0.683 & 0.463 & 0.700 \\
\hline & 2 & -0.017 & -0.695 & 0.631 & 0.923 \\
\hline & 3 & -0.059 & -0.768 & 0.543 & 0.730 \\
\hline & 4 & -0.087 & -0.851 & 0.519 & 0.626 \\
\hline
\end{tabular}

\section{Discussion}

Hypertriglyceridemia is a highly prevalent metabolic disorder which arises as a result of secondary conditions such as high fat diet or definite inherited basis such as lipoprotein lipase gene deficiency [6]. Severe cases of hypertriglyceridemia, that is SHTG, are presented in approximately $1.7 \%$ of these adult population and about $15-20 \%$ of this group develop sHTGP [5]. It is widely accepted that the increase in 
serum TG levels has a positive correlation with disease severity and AP recurrence [12]. As regards disease severity, $23.4 \%$ sHTGP patients developed SAP in our study and the proportion was only $9.6 \%$ in whole HTG-induced pancreatitis cohort as reported previously [16]. The current reported recurrence rate of HTG-induced pancreatitis was about $25-30 \%$, while the recurrence of sHTGP was about $50 \%$ in our study $[16,17]$. A longitudinal cohort study of patients with HTG-induced pancreatitis demonstrated that even moderate elevation in subsequent TG levels was associated with increased risk of recurrence compared to patients that achieved normalization (RR $5.47[1.80,16.65]$ ) [18]. It is generally considered that patients with HTG are often complicated with metabolic abnormalities such as diabetes [6]. $36.1 \%$ sHTGP patients in our cohort were complicated with diabetes and the proportion was higher in the recurrence group. A multivariate analysis had illustrated that presence of diabetes was an independent risk factor for AP recurrence [19].

In our study, we found that the lipid profile of sHTGP changed obviously after DFPP in the first day and the level of parameters maintained stability except for serum TG. Compared with other parameters of the lipid profile, TG was the most intense and variable parameter during the explosion of SHTGP. All the patients resumed oral feeding at discharge and the level of parameters at this point may serve as the normal reference. Interestingly, the levels of lipid profile at disease onset exhibited significant difference from the normal level of patients, indicating the burst of sHTGP may be concerned with an acute and dramatic fluctuation of the lipid metabolism. The results conform to the prevalent speculations for the pathogenesis of SHTGP [8].

It is generally accepted that rapid decrease in TG levels is critical to the successful management of HTGinduced pancreatitis [20]. However, the pharmacologic dyslipidemia therapy such as insulin and heparin was insufficient to lowering TG rapidly [21]. In contrast, apheresis can rapidly reduce serum chylomicron and TG levels. According to a systemic review published in 2017, apheresis can reduce the pre-treatment serum TG (mean: $42.0 \mathrm{mmol} / \mathrm{L}$ ) by $72 \%$. As a therapeutic plasmapheresis which can remove macromolecules selectively, DFPP has been applied for sHTGP management in recent years. Our results showed similar efficiency of TG lowering effect with previous studies (pretreatment TG: $42.68 \mathrm{mmol} / \mathrm{L}$ by $71.2 \%)$. There were few studies exploring the effect of DFPP on sHTGP management. Chang et al. investigated the effectiveness of DFPP in twelve sHTGP patients and found that DFPP could shorten hospitalization duration and minimizes AP recurrence. In our study, we found that sHTGP patients in which TG level reached below $5.6 \mathrm{mmol} / \mathrm{L}$ after DFPP had shorter hospitalization duration [22].

Lipids and lipoproteins undergo changes during systemic inflammatory response syndrome (SIRS) and may act as potential biomarkers (especially HDL) [23]. It was observed that critically ill patients showed low concentrations of HDL and Apo A1 upon ICU admission which correlated with increased severity, ICU duration and mortality $[24,25]$. Similar to findings in previous studies, we found that decreased HDL appears to be indicative of the extension of SHTGP hospitalization $[11,26]$. HDL is the complex of lipoprotein species which contain approximately $25 \%$ of the cholesterol and $<5 \%$ of the triglyceride in human blood [27]. They play a major role in TG transport and removal after lipase action. It is generally considered that HDL has potent anti-inflammatory properties which may be critical for protection against 
AP and other inflammatory disease through modulating macrophages reprogramming $[11,28]$. Lower levels of HDL were elucidated to be associated with increased cardiovascular events and poor outcomes $[29,30]$. Bugdaci et al. reported that the levels of HDL were negatively associated with the Ranson score of AP [26]. Khan et al. found that levels of serum HDL was significantly lower in SAP patients and was associated with longer hospitalization [11].

There were several limitations of this study. First, this was a retrospective analysis and we included relatively small number of patients due to the single center design. Second, this study lacks the control group for comparison with the DFPP group. In the real-world practice, all the sHTGP patients received DFPP on admission in our center and consequently the study lacks untreated sHTGP cohort. Further large scale and multicenter studies are needed.

\section{Conclusions}

In summary, there was distinct fluctuation of the lipid profile upon the burst of sHTGP and the parameters changed significantly in the first day after DFPP. Among these parameters, HDL may serve as a biomarker of disease prognosis in patients with sHTGP. Moreover, reaching the TG-lowering target of $5.6 \mathrm{mmol} / \mathrm{L}$ may shorten hospitalization duration.

\section{Abbreviations}

AP, acute pancreatitis; APACHE II, Acute Physiology and Chronic Health Evaluation score II; Apo A1, Apoprotein A1; BMI, body mass index; DFPP, double filtration plasmapheresis; HDL, high density lipoprotein; ICU, intensive care unit; LDL, low density lipoprotein; SHTG, severe hypertriglyceridemia; sHTGP, severe hypertriglyceridemia-induced acute pancreatitis; SAP, severe acute pancreatitis; SOFA, sequential organ failure assessment score; TC, total cholesterol; VLDL, very low density lipoprotein.

\section{Declarations}

\section{Ethics approval and consent to participate}

The study was approved by the Ethics Committee of Sir Run Run Shaw Hospital, Zhejiang University School of Medicine (20190215-3). Because of the retrospective nature of this study, informed consent was waived.

\section{Consent for publication}

All authors provide consent for publication of this paper.

\section{Availability of data and materials}

All data generated and analyzed in this study are included in this published article. The datasets are available from the corresponding author on reasonable request. 


\section{Competing interests}

All authors of this paper have no competing interests to disclose.

\section{Funding}

This study is supported by the Natural Science Foundation of Zhejiang Province (LQ2OH030011) and the Medical Health Science and Technology Project of Zhejiang Provincial Health Commission (2020386600).

\section{Authors' contributions}

The study was designed by Na Yang and Feng Guo; Donghai Wang, Huijun Zheng, Xiaoling Wang and Yongjun Lin analyzed and interpreted the data; Huijun Zheng and Zhihua Lu were responsible for drafting the manuscript; The manuscript was reviewed by Yueliang Chen. All authors have read and approved the final manuscript for publication.

\section{Acknowledgements}

Not applicable.

\section{Author details}

${ }^{1}$ Department of Critical Care Medicine, Sir Run Run Shaw Hospital, Zhejiang University School of Medicine, Hangzhou 310016, Zhejiang, China.

\section{References}

1. Garg SK, Sarvepalli S, Campbell JP, Obaitan I, Singh D, Bazerbachi F, Singh R, Sanaka MR. Incidence, Admission Rates, and Predictors, and Economic Burden of Adult Emergency Visits for Acute Pancreatitis: Data From the National Emergency Department Sample, 2006 to 2012. J Clin Gastroenterol. 2019;53:220-5.

2. Yadav D, Lowenfels AB. The epidemiology of pancreatitis and pancreatic cancer. Gastroenterology. 2013;144:1252-61.

3. Ewald N, Hardt PD, Kloer HU. Severe hypertriglyceridemia and pancreatitis: presentation and management. Curr Opin Lipidol. 2009;20:497-504.

4. Zhu Y, Pan X, Zeng H, He W, Xia L, Liu P, Zhu Y, Chen Y, Lv N. A Study on the Etiology, Severity, and Mortality of 3260 Patients With Acute Pancreatitis According to the Revised Atlanta Classification in Jiangxi, China Over an 8-Year Period. Pancreas. 2017;46:504-9.

5. Adiamah A, Psaltis E, Crook M, Lobo DN. A systematic review of the epidemiology, pathophysiology and current management of hyperlipidaemic pancreatitis. Clin Nutr. 2018;37:1810-22. 
6. Lewis GF, Xiao C, Hegele RA. Hypertriglyceridemia in the genomic era: a new paradigm. Endocr Rev. 2015;36:131-47.

7. Mosztbacher D, Hanák L, Farkas N, Szentesi A, Mikó A, Bajor J, Sarlós P, Czimmer J, Vincze Á, Hegyi PJ, et al. Hypertriglyceridemia-induced acute pancreatitis: A prospective, multicenter, international cohort analysis of 716 acute pancreatitis cases. Pancreatology. 2020;20:608-16.

8. Pedersen SB, Langsted A, Nordestgaard BG. Nonfasting Mild-to-Moderate Hypertriglyceridemia and Risk of Acute Pancreatitis. JAMA Intern Med. 2016;176:1834-42.

9. Zhang Q, Qin M, Liang Z, Huang H, Tang Y, Qin L, Wei Z, Xu M, Tang G. The relationship between serum triglyceride levels and acute pancreatitis in an animal model and a 14-year retrospective clinical study. Lipids Health Dis. 2019;18:183.

10. Wu Q, Zhong X, Fu M, Yang H, Bo H, Liao X, Hu Z, Wang B, Zhang Z, Jin X, Kang Y. High-density lipoprotein cholesterol to low-density lipoprotein cholesterol ratio in early assessment of disease severity and outcome in patients with acute pancreatitis admitted to the ICU. BMC Gastroenterol. 2020;20:164.

11. Khan J, Nordback I, Sand J. Serum lipid levels are associated with the severity of acute pancreatitis. Digestion. 2013;87:223-8.

12. Scherer J, Singh VP, Pitchumoni CS, Yadav D. Issues in hypertriglyceridemic pancreatitis: an update. J Clin Gastroenterol. 2014;48:195-203.

13. Galán Carrillo I, Demelo-Rodriguez P, Rodríguez Ferrero ML, Anaya F. Double filtration plasmapheresis in the treatment of pancreatitis due to severe hypertriglyceridemia. J Clin Lipidol. 2015;9:698-702.

14. Nakanishi T, Suzuki N, Kuragano T, Nagasawa Y, Hasuike Y. Current topics in therapeutic plasmapheresis. Clin Exp Nephrol. 2014;18:41-9.

15. Banks PA, Bollen TL, Dervenis C, Gooszen HG, Johnson CD, Sarr MG, Tsiotos GG, Vege SS. Classification of acute pancreatitis-2012: revision of the Atlanta classification and definitions by international consensus. Gut. 2013;62:102-11.

16. Kim SJ, Kang H, Kim EJ, Kim YS, Cho JH. Clinical features and outcomes of hypertriglyceridemiainduced acute pancreatitis: Propensity score matching analysis from a prospective acute pancreatitis registry. Pancreatology. 2020;20:617-21.

17. Gillies NA, Pendharkar SA, Singh RG, Asrani VM, Petrov MS. Lipid metabolism in patients with chronic hyperglycemia after an episode of acute pancreatitis. Diabetes Metab Syndr. 2017;11(Suppl 1):233-s241.

18. Wu BU, Batech M, Dong EY, Duan L, Yadav D, Chen W. Influence of Ambulatory Triglyceride Levels on Risk of Recurrence in Patients with Hypertriglyceridemic Pancreatitis. Dig Dis Sci. 2019;64:890-7.

19. Zafrir B, Saliba W, Jubran A, Hijazi R, Shapira C. Severe Hypertriglyceridemia-Related Pancreatitis: Characteristics and Predictors of Recurrence. Pancreas. 2019;48:182-6.

20. de Pretis N, Amodio A, Frulloni L. Hypertriglyceridemic pancreatitis: Epidemiology, pathophysiology and clinical management. United European Gastroenterol J. 2018;6:649-55. 
21. Dhindsa S, Sharma A, Al-Khazaali A, Sitaula S, Nadella S, McKee A, Albert S, Bourey R, Dandona P. Intravenous Insulin Versus Conservative Management in Hypertriglyceridemia-Associated Acute Pancreatitis. J Endocr Soc. 2020;4:bvz019.

22. Chang CT, Tsai TY, Liao HY, Chang CM, Jheng JS, Huang WH, Chou CY, Chen CJ. Double Filtration Plasma Apheresis Shortens Hospital Admission Duration of Patients With Severe Hypertriglyceridemia-Associated Acute Pancreatitis. Pancreas. 2016;45:606-12.

23. Wendel M, Paul R, Heller AR. Lipoproteins in inflammation and sepsis. II. Clinical aspects. Intensive Care Med. 2007;33:25-35.

24. Golucci A, Marson FAL, Ribeiro AF, Nogueira RJN. Lipid profile associated with the systemic inflammatory response syndrome and sepsis in critically ill patients. Nutrition. 2018;55-56:7-14.

25. Cabana VG, Siegel JN, Sabesin SM. Effects of the acute phase response on the concentration and density distribution of plasma lipids and apolipoproteins. J Lipid Res. 1989;30:39-49.

26. Bugdaci MS, Sokmen M, Zuhur SS, Altuntas Y. Lipid profile changes and importance of low serum alipoprotein fraction (high-density lipoprotein) in cases with acute pancreatitis. Pancreas. 2011;40:1241-4.

27. Brown WV. High-density lipoprotein and transport of cholesterol and triglyceride in blood. J Clin Lipidol. 2007;1:7-19.

28. De Nardo D, Labzin LI, Kono H, Seki R, Schmidt SV, Beyer M, Xu D, Zimmer S, Lahrmann C, Schildberg FA, et al. High-density lipoprotein mediates anti-inflammatory reprogramming of macrophages via the transcriptional regulator ATF3. Nat Immunol. 2014;15:152-60.

29. Nakazawa M, Arashi H, Yamaguchi J, Ogawa H, Hagiwara N. Lower levels of high-density lipoprotein cholesterol are associated with increased cardiovascular events in patients with acute coronary syndrome. Atherosclerosis. 2020;303:21-8.

30. Hu J, Xi D, Zhao J, Luo T, Liu J, Lu H, Li M, Xiong H, Guo Z. High-density Lipoprotein and Inflammation and Its Significance to Atherosclerosis. Am J Med Sci. 2016;352:408-15.

\section{Figures}


a

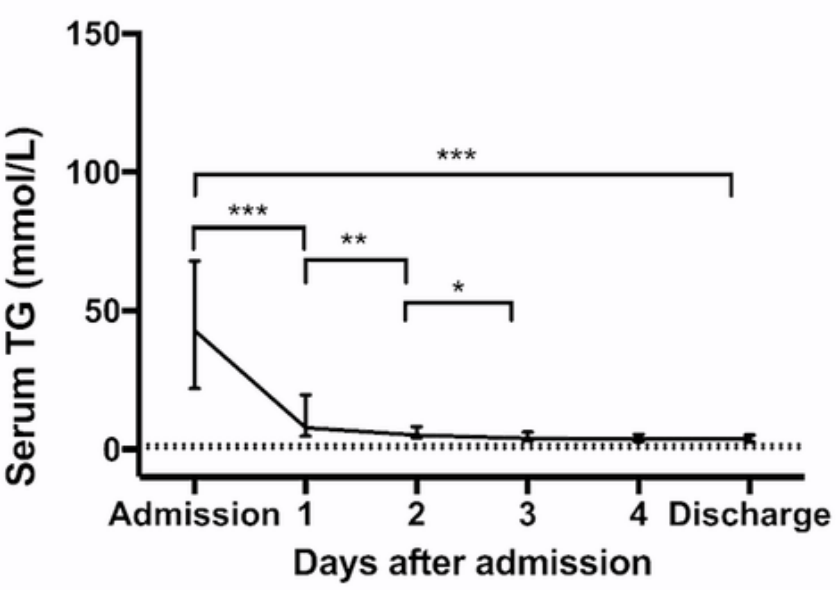

C

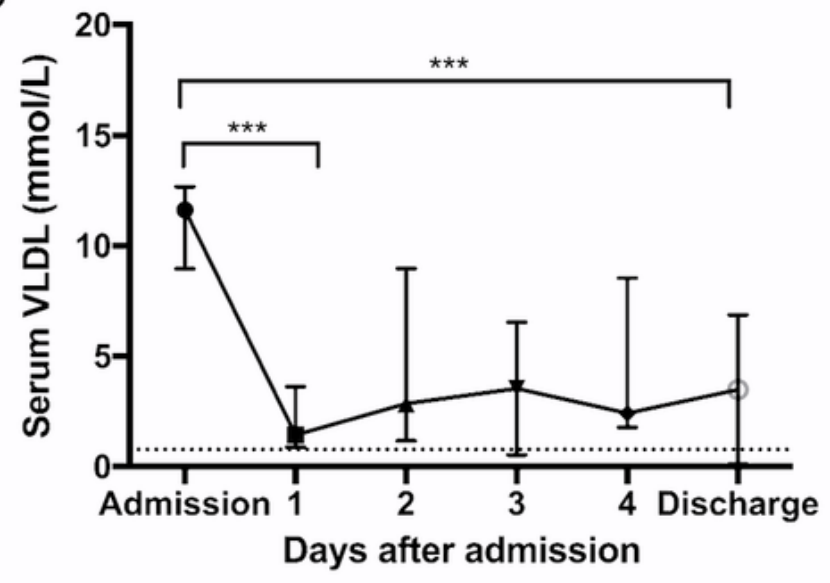

e

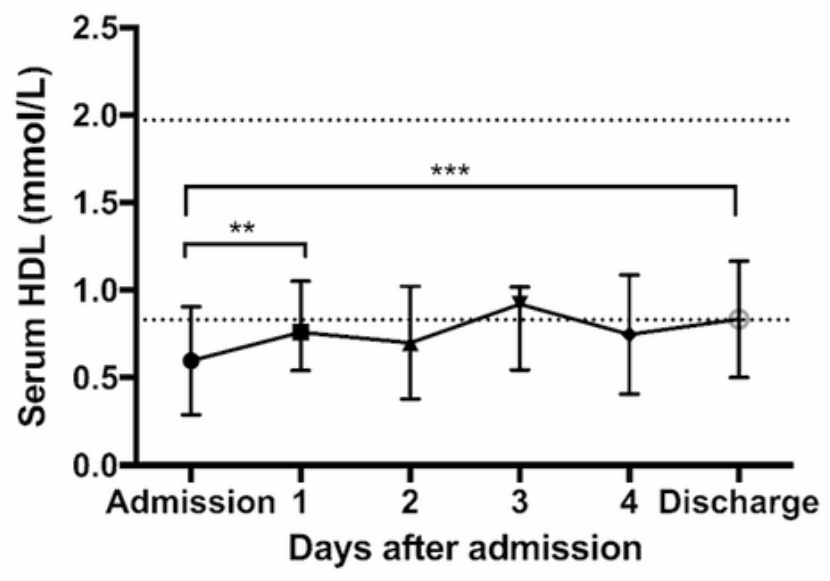

b

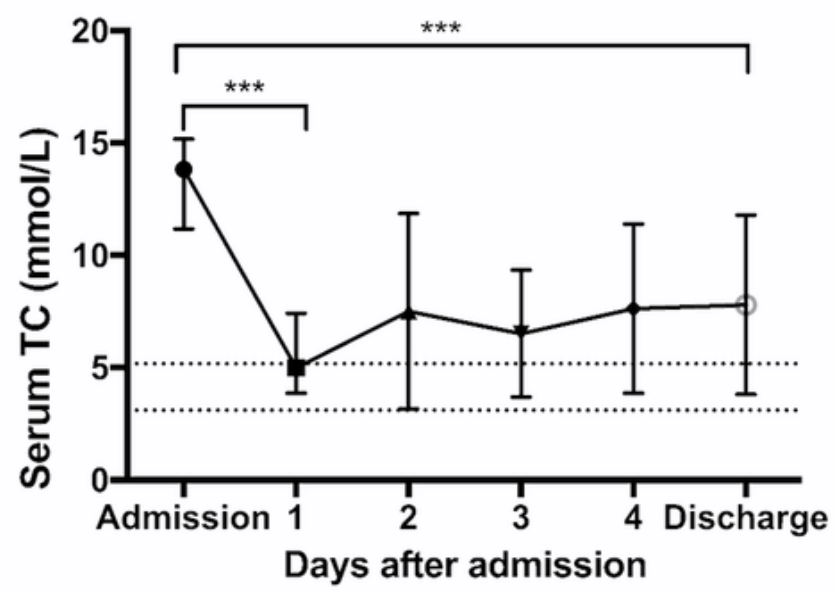

$\mathrm{d}$

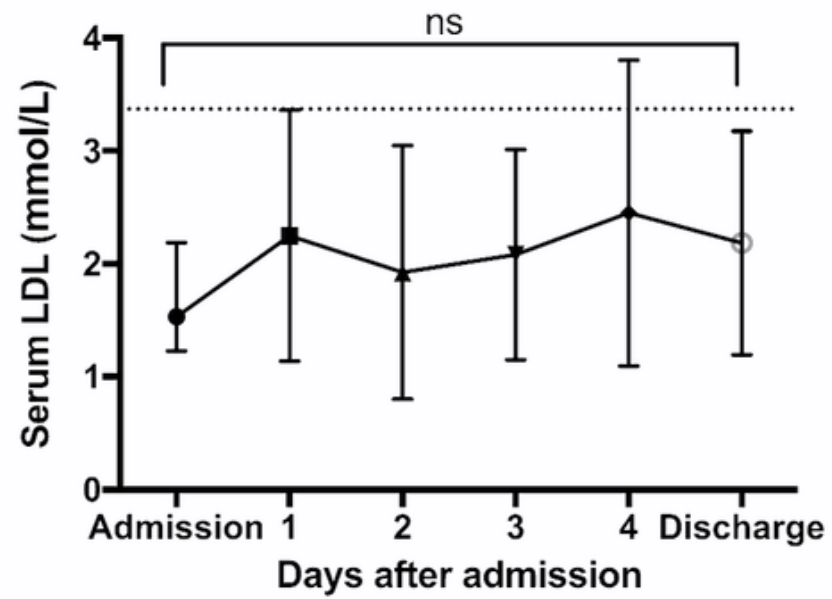

f

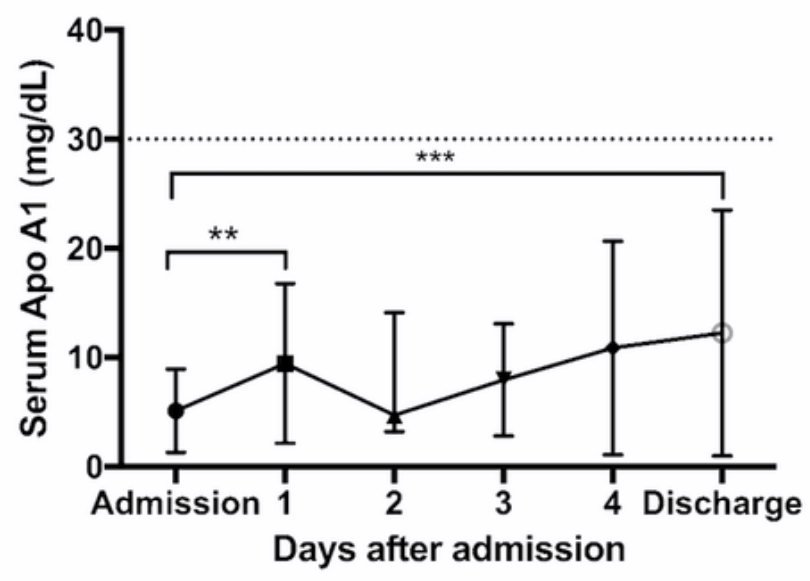

Figure 1

Dynamic changes of lipid profile before and after DFPP during SHTGP. Data are presented as medians, with I bars indicating interquartile ranges. ${ }^{*} P<0.05$, ${ }^{\star} \mathrm{P}<0.01,{ }^{\star} * * P<0.001$. ns, no sense. The dotted lines represent the reference standard of these parameters in our center. 